\title{
Meteorological uncertainty and rainfall downscaling
}

\author{
J. von Hardenberg ${ }^{1}$, L. Ferraris ${ }^{2}$, N. Rebora ${ }^{2}$, and A. Provenzale ${ }^{1}$ \\ ${ }^{1}$ ISAC-CNR, Corso Fiume 4, 10133 Torino, Italy \\ ${ }^{2}$ CIMA - Università di Genova, via Cadorna 7, 17100 Savona, Italy
}

Received: 12 January 2007 - Revised: 17 April 2007 - Accepted: 4 May 2007 - Published: 22 May 2007

\begin{abstract}
We explore the sources of forecast uncertainty in a mixed dynamical-stochastic ensemble prediction chain for small-scale precipitation, suitable for hydrological applications. To this end, we apply the stochastic downscaling method RainFARM to each member of ensemble limitedarea forecasts provided by the COSMO-LEPS system. Aim of the work is to quantitatively compare the relative weights of the meteorological uncertainty associated with large-scale synoptic conditions (represented by the ensemble of dynamical forecasts) and of the uncertainty due to small-scale processes (represented by the set of fields generated by stochastic downscaling). We show that, in current operational configurations, small- and large-scale uncertainties have roughly the same weight. These results can be used to pinpoint the specific components of the prediction chain where a better estimate of forecast uncertainty is needed.
\end{abstract}

\section{Introduction}

Operational assessment of flood risk in small basins, with areas up to a few hundred square kilometers (such as those encountered in mountainous regions and along the Mediterranean coast), requires detailed precipitation forecasts on small spatial and temporal scales (Ferraris et al., 2002), as well as estimates of the associated prediction uncertainty. However, the smallest spatial scales reliably resolved by regional meteorological models, used for real-time ensemble predictions, rarely go below the size of these basins. In addition, computational limitations allow for regional meteorological ensembles with a relatively small number of members (not larger than about twenty).

An alternative approach to cope with small-scale ensemble predictions resorts to the use of stochastic models for rainfall

Correspondence to: J. von Hardenberg

(j.vonhardenberg@isac.cnr.it) downscaling (see e.g. Ferraris et al., 2003, for a comparison of different approaches). In this framework, an ensemble of small-scale stochastic rainfall fields with prescribed statistical properties, usually defined from larger-scale meteorological forecasts, is used to estimate the probability of occurrence of intense rainfall events. When the stochastic fields are fed into a rainfall-runoff model of selected basins, this method allows for estimating the probability of flood occurrence (Ferraris et al., 2002). Operational assessment of the probability of extreme rainfall events and of their uncertainty should thus be based on a mixed dynamical-stochastic rainfall forecasting chain, based on two main ingredients (Siccardi et al., 2005): (1) an ensemble of limited-area meteorological forecasts, used to capture the dynamical variability and the sensitivity to initial conditions of the atmosphere; (2) from each of these forecasts, the generation of an ensemble of rainfall fields produced by a stochastic downscaling model.

In this type of approach, the stochastic downscaling procedure plays two roles. On one hand, it allows for estimating the probability of occurence of intense precipitation on small spatial and temporal scales. On the other hand, it also introduces additional variability which "dresses" individual dynamical ensemble forecasts (Roulston and Smith, 2003), compensating for the usually very small number of dynamical ensemble members in meteorological forecasts.

The present article focusses on the second issue. In this work, we analyse for the first time the practical construction of a mixed dynamical-stochastic ensemble prediction chain and compare the large-scale uncertainty represented by the dynamical meteorological ensemble with that modelled by the use of stochastic downscaling at small scales. Ensemble meteorological predictions are provided by the COSMOLEPS limited area ensemble prediction system (Molteni et al., 2001; Montani et al., 2001, 2003; Marsigli et al., 2001, 2004,2005 ), and the downscaling procedure is based on the approach discussed by Rebora et al. (2006a,b). Both

Published by Copernicus GmbH on behalf of the European Geosciences Union and the American Geophysical Union. 
procedures have been shown to provide well calibrated, probabilistic forecasts of precipitation at large and small scales respectively. The results of the analysis will be used to provide some general guidelines on how to determine, in an empirical way, the specific components of the prediction chain where a better assessment of the forecast uncertainty leads to significant improvement of the overall uncertainty estimates.

\section{Uncertainty and ensemble predictions}

Every meteorological forecast is invariably affected by uncertainity: Measurement error and finite resolution in the initial conditions, incomplete representation of the physics of the problem and finite numerical resolution, corresponding to an incomplete representation of subgrid processes, all lead to an uncertainity in the forecast which grows with forecast time and rapidly propagates to all scales (see e.g. Palmer, 2000). Dynamical ensemble forecasting (Epstein, 1969; Leith, 1974; Toth and Kalnay, 1993; Palmer, 1993; Molteni et al., 1996; Toth and Kalnay, 1997) represents the current method of choice for taking into account some of these sources of uncertainity, by estimating the probability of occurrence of different meteorological scenarios from the relative frequency of different forecast ensemble members. Current computational constraints limit the resolution of operational global ensemble forecasts to spatial scales of at best $50 \mathrm{~km}$ and to few tens of ensemble members (the operational ECMWF ensemble with its 51 members is one of the largest ones). Stochastic perturbations of the model physics and a multi-model approach can be used to take into account model error (Palmer, 2001).

At a regional level, global ensemble forecasts can be extended to fine scales (currently around $10 \mathrm{~km}$ ), either by independent integration on limited areas or by nesting high resolution, often non-hydrostatic, limited area models (LAM) in the general circulation scenarios. Examples of such systems are COSMO-LEPS, HIRLAM-LAMEPS (Frogner and Iversen, 2002; Sattler and Feddersen, 2005) and the NCEP SREF system (Hamill and Colucci, 1997, 1998; Du et al., 1997). However, due to the incomplete representation of small scale processess, to the limited resolution of the observational network used for initialization and data assimilation, and to numerical details (Adlerman and Droegemeier, 2002), currently these models have low prediction skill already at spatial scales of a few tens of kilometers, even if their nominal resolution is higher. Also for LAMs, computational constraints severely limit the number of ensemble members, and ensemble size has been shown to have a significant impact on performance statistics in these systems (Marsigli et al., 2005).

Both at large and small scales, the limited size of the ensembles is associated with a significant residual error in the forecast, due to undersampling of the forecast probability distribution function (Buizza and Palmer, 1998). This er- ror can become severe when small regional areas are considered, leading to significant mistakes in the estimates of the probability of extreme precipitation events. Roulston and Smith (2003) suggested to take into account the residual errors in the forecasts by creating stochastic "daughter" ensembles which dress each ensemble member with a statistical error ensemble. With such methods, large stochastic ensembles can be easily generated and a smoother representation of future probability distributions can be obtained, but the resulting spatio-temporal fields do not necessarily preserve the statistical properties of the observed fields. As such, this approach is not suitable for hydrological applications, where spatial and temporal correlations are important.

An alternative approach is based on the construction of stochastic rainfall prediction ensembles, using downscaling methods (Ferraris et al., 2002). From a single deterministic forecast, these methods allow for the generation of large ensembles of higher resolution, stochastic, spatiotemporal fields, which share the desired (larger-scale) statistical properties with the original meteorological forecast. The small scale properties of precipitation in these models are purely stochastic and simply obtained by propagating the large-scale information in the forecast. In past years, several downscaling approaches have been proposed, including point-process models (see e.g. Waymire et al., 1984), autoregressive models (e.g. Bell, 1987), and fractal cascades (e.g. Lovejoy and Mandelbrot, 1985; Perica and FoufoulaGeorgiu, 1996; Menabde et al., 1997), all of which have similar skills in reproducing the statistical properties of observed precipitation (Ferraris et al., 2003).

\section{Sources of uncertainty in a precipitation prediction chain}

In this work, we apply a stochastic downscaling procedure to each member of an ensemble of dynamical forecasts generated by the COSMO-LEPS limited-area ensemble prediction system with spatial resolution $10 \mathrm{~km}$. The study area is a square region of $320 \mathrm{~km}$ on each side, centered over northwestern Italy, see Fig. 1a. For the purpose of this work, we chose four study cases of intense precipitation events (forecasts issued on 13 November 2002, 23 November 2002, 26 December 2002 and 20 January 2003). For each case, the COSMO-LEPS system operational in 2002/2003 produced an ensemble of $N_{L}=5$ forecast fields, issued at noon every day, with a total forecast time of $120 \mathrm{~h}$. The precipitation forecasts are cumulated over a period of $6 \mathrm{~h}$; Fig. 1a also shows an example LEPS forecast member.

The dynamically forecasted fields are downscaled to finer resolution using the stochastic model RainFARM (Rebora et al., 2006b). This model is based on a nonlinear transformation of a linearly-correlated stochastic field, obtained by extrapolation to small scales of the large-scale power spectrum of the forecast. A power law functional form is assumed 

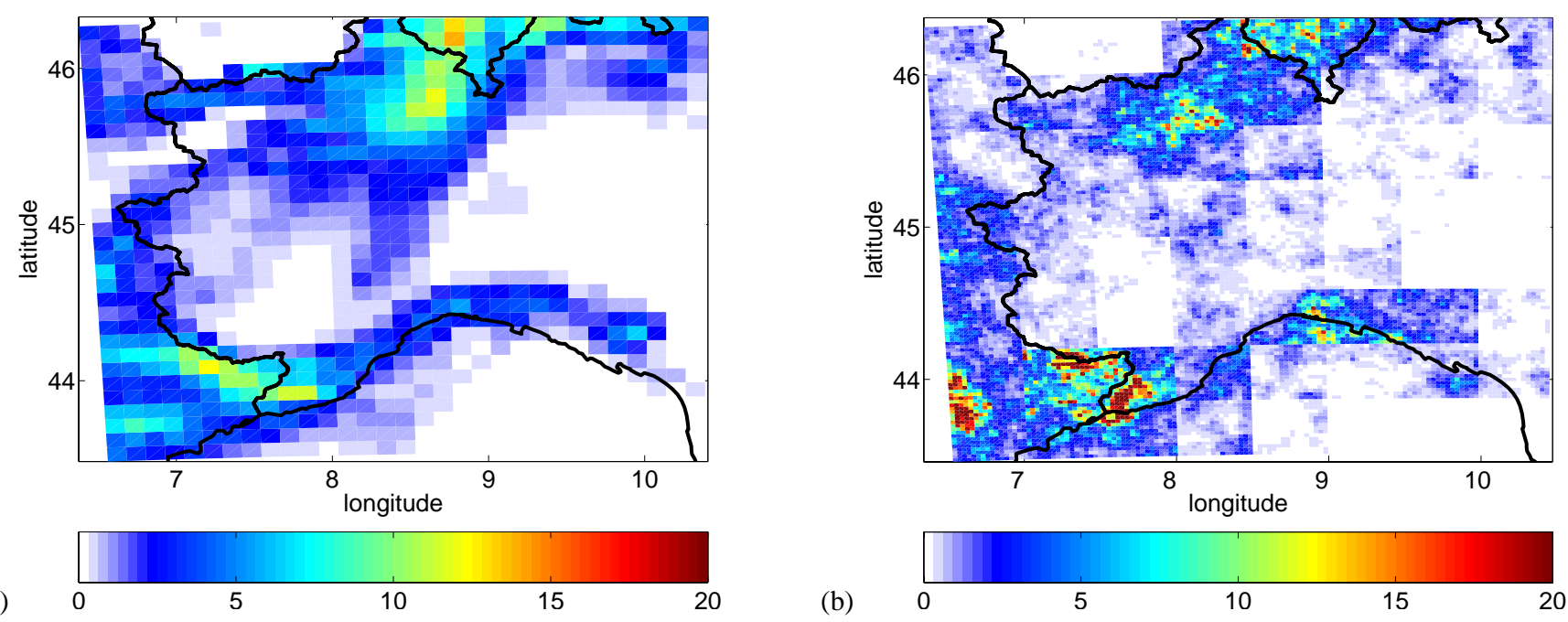

Fig. 1. The computational domain considered in this work, centered on north-western Italy, together with (a) a snapshot of a LEPS precipitation forecast with spatial resolution $10 \mathrm{~km}$; and (b) the same field downscaled with RainFARM, using $L_{O}=40 \mathrm{~km}$ and $T_{O}=6 \mathrm{~h}$. Both panels refer to the forecast issued on 13 November 2002 at forecast time $\tau=12 \mathrm{~h}$. Precipitation is expressed in units of [mm/h] and averaged over $6 \mathrm{~h}$ in panel (a) and over $24 \mathrm{~min}$ in panel (b).

for the extrapolation and random Fourier phases are used at the unresolved scales. The downscaled fields produced by this model are easily nested in regional-scale meteorological forecasts. The rain fields generated by the downscaling procedure are characterized by stochastic variability only at scales smaller than the "reliability scales", $L_{o}$ and $T_{o}$, and preserve the larger-scale properties of the member of the dynamical ensemble from which they are derived. These reliability scales represent the (empirically determined) scales below which the dynamical forecasts lose their predictive power; usually, $L_{o}$ is about 4 to 6 times the nominal resolution of the model (Patterson and Orszag, 1971). The model produces fields which, when aggregated at scales larger than $L_{o}$ and $T_{o}$, preserve total precipitation volumes, the location of large-scale structures, the spatial and temporal correlations of the original fields and the multifractal properties of the precipitation fields. A detailed verification of the properties of the fields generated by RainFARM is discussed in Rebora et al. (2006a,b).

The reliability scales $L_{o}$ and $T_{o}$ represent the only free parameters of the model, since the other important parameters, such as the spectral slopes used to extrapolate the power spectrum to small scales, are derived from the largescale properties of the precipitation field to be downscaled. With this procedure, for each member of the LEPS ensemble we generate a stochastic ensemble of $N_{R}=100$ downscaled fields with spatial resolution $\Delta x=2.5 \mathrm{~km}$ and temporal resolution of $\Delta t=24 \mathrm{~min}$. By construction, each of these fields is identical to the original meteorological forecast when aggregated on the reliability scales. In the following, we explore different values for the reliability scales, and consider
$L_{o}=[20,40,80,160] \mathrm{km}$ and $T_{o}=[6,12] \mathrm{h}$. As an example, Fig. 1b shows a snapshot of one field obtained by downscaling the LEPS forecast of Fig. 1a.

In the following, we indicate with $\mathbf{p}_{i k}(x, y, t)$ the $k$-th member $\left(k=1 \ldots N_{R}\right)$ of the ensemble of stochastic precipitation fields generated by downscaling, with $i=1 \ldots N_{L}$ indicating the member of the LEPS ensemble to which the downscaling procedure was applied. The position in space is $(x, y)$ and $t$ is time. Each precipitation field can be thought of as a vector in a high-dimensional space whose axes are defined by all spatial positions and temporal instants spanned by the field. From each stochastic ensemble, we derive the ensemble mean, $\overline{\mathbf{p}}_{i}(x, y, t)=\frac{1}{N_{R}} \sum_{k=1}^{N_{R}} \mathbf{p}_{i k}(x, y, t)$. We characterize the spread of the $i$-th stochastic ensemble using its r.m.s. deviation around the mean: $\sigma_{i}^{2}=\frac{1}{N_{R}} \sum_{k=1}^{N_{R}}\left|\mathbf{p}_{i k}-\overline{\mathbf{p}}_{i}\right|^{2}$, where the operator $\|^{2}$ indicates the mean quadratic distance beetween two spatio-temporal fields.

To quantify the distance between two stochastic ensembles generated from two different LEPS fields, we determine the distances between their centers: $D_{i j}^{2}=\left|\overline{\mathbf{p}}_{i}-\overline{\mathbf{p}}_{j}\right|^{2}$, from which we derive the average distance of each stochastic ensemble from the others as $S_{i}^{2}=\frac{1}{\left(N_{L}-1\right)} \sum_{j \neq i} D_{i j}^{2}$. We further define a measure of the average spread of stochastic ensembles, $\bar{\sigma}^{2}=\frac{1}{N_{L}} \sum_{i=1}^{N_{L}} \sigma_{i}^{2}$, and an average spread of each dynamical ensemble, $\bar{S}^{2}=\frac{1}{N_{L}} \sum_{i=1}^{N_{L}} S_{i}^{2}$. The different objects and some of the measures defined above are illustrated schematically in Fig. 2, which shows how each LEPS member, represented by crosses, is associated with a corresponding stochastic ensemble. 


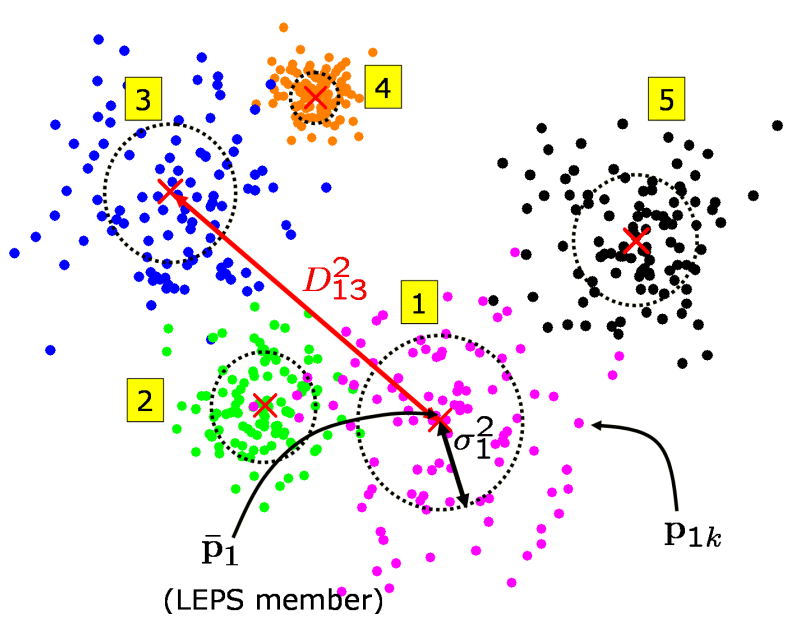

Fig. 2. An idealized illustration of the statistical quantities considered in the analysis, obtained by projecting onto a two-dimensional plane the high-dimensional space spanned by the precipitation fields. For each of the five LEPS ensemble members, a set of 100 stochastic rain fields were generated using RainFARM (the "stochastic ensembles"). The width of each stochastic ensemble is measured by its variance and is indicated by the dashed circles in the figures. The distances between ensemble centers, indicated by crosses, measure the spread of the dynamical LEPS ensemble. The figure indicates, as an example, the distance between the centers of the stochastic ensembles obtained by downscaling the first and third members of the LEPS ensemble.

Armed with these definitions, we now compare the spread of each stochastic ensemble, $\sigma_{i}^{2}$, with its average distance from other stochastic ensembles, $S_{i}^{2}$, for different values of the spatial and temporal reliability scales and for the four events considered. The statistics represented in the following figures are based on the spatio-temporal RMS distance between the downscaled fields. Spatial and temporal dependence appear in the choice of the reliability scales. Figure 3 shows that, for most of the events considered, variations in the reliability scales do not significantly affect the spread of individual stochastic ensembles. Note that increasing the reliability scales leads to a reduction in $S_{i}^{2}$. Indeed, by construction of the RainFARM procedure, the center of each stochastic ensemble roughly corresponds to the field obtained by aggregating the original meteorological forecast at the reliability scales. Increasing $L_{o}$ and $T_{o}$ thus leads to a reduction of the average distance between the stochastic ensemble centers.

When $L_{o}$ and $T_{o}$ are small, the spread of each stochastic ensemble typically stays well below the average distance between the ensemble centers. By contrast, for large values of $L_{o}$ and $T_{o}$, the spread of the individual stochastic ensembles can become larger than the average distance between the ensemble centers. One of the four cases (the forecast issued on 13 November 2002) represents an exception: the spread of the stochastic ensembles exceeds the average distance between the ensemble centers for all choices of the reliability scales. What distinguishes this case from the others is a particularly large variance of the LEPS member fields themselves, which in RainFARM leads to a large spread of the stochastic ensembles.

The ratio of the average spread of the stochastic ensembles to that of the dynamical ensembles, $\bar{\sigma}^{2} / \bar{S}^{2}$, gauges the relative importance of these two components of total variability and is reported in Fig. 4 as a function of the reliability scales. There is a significant dependence of this ratio on the reliability scales. For most events, stochastic variability becomes larger than the dynamic one as reliability scales cross from 40 to $80 \mathrm{~km}$ (if $T_{o}=6 \mathrm{~h}$ ) or from 20 to $40 \mathrm{~km}$ (if $T_{o}=12 \mathrm{~h}$ ), with the only exception of the forecast issued on 13 November 2002 .

\section{Discussion and conclusions}

This work can be considered a first step towards the practical construction of an operational mixed dynamical-stochastic forecasting chain for the management of hydrogeological risk. In particular, we compared the uncertainty associated with stochastic downscaling with the uncertainity present in a meteorological ensemble, in order to obtain a better quantification of the prediction uncertainty in small-scale rainfall prediction.

In order for stochastic downscaling to be a useful element of the prediction chain, the additional variability introduced by the downscaling procedure should be of the same order of the spread of the dynamical ensemble. When the spread of the dynamical ensemble is significantly larger than smallscale stochastic variability, the downscaling procedure can lead only to a marginal advantage in bracketing observations; viceversa a very large stochastic variability with respect to dynamical ensemble spread could completely swamp out the information provided by the dynamical ensemble.

The results obtained here show that the ratio between the variability introduced by small-scale stochastic downscaling and that associated with the dynamical ensemble at larger scales depends on the value of the smallest scales above which the meteorological predictions are considered reliable. The best choice of these scales for the COSMO-LEPS system is currently uncertain. Nonetheless, numerical diffusion problems usually make numerical models unreliable at resolutions which are below 4/6 times their grid resolution (Patterson and Orszag, 1971). Using this criterion, we can approximately estimate the spatial reliability scale of COSMOLEPS forecasts as $L_{o}=40 / 60 \mathrm{~km}$. Figures 3 and 4 show that for three of the four events considered, this choice of reliability scales leads to dynamical and stochastic ensembles which contribute almost equally to the total variability of the forecast. Thus, the proposed operational forecasting chain can be considered well equilibrated, since both components 

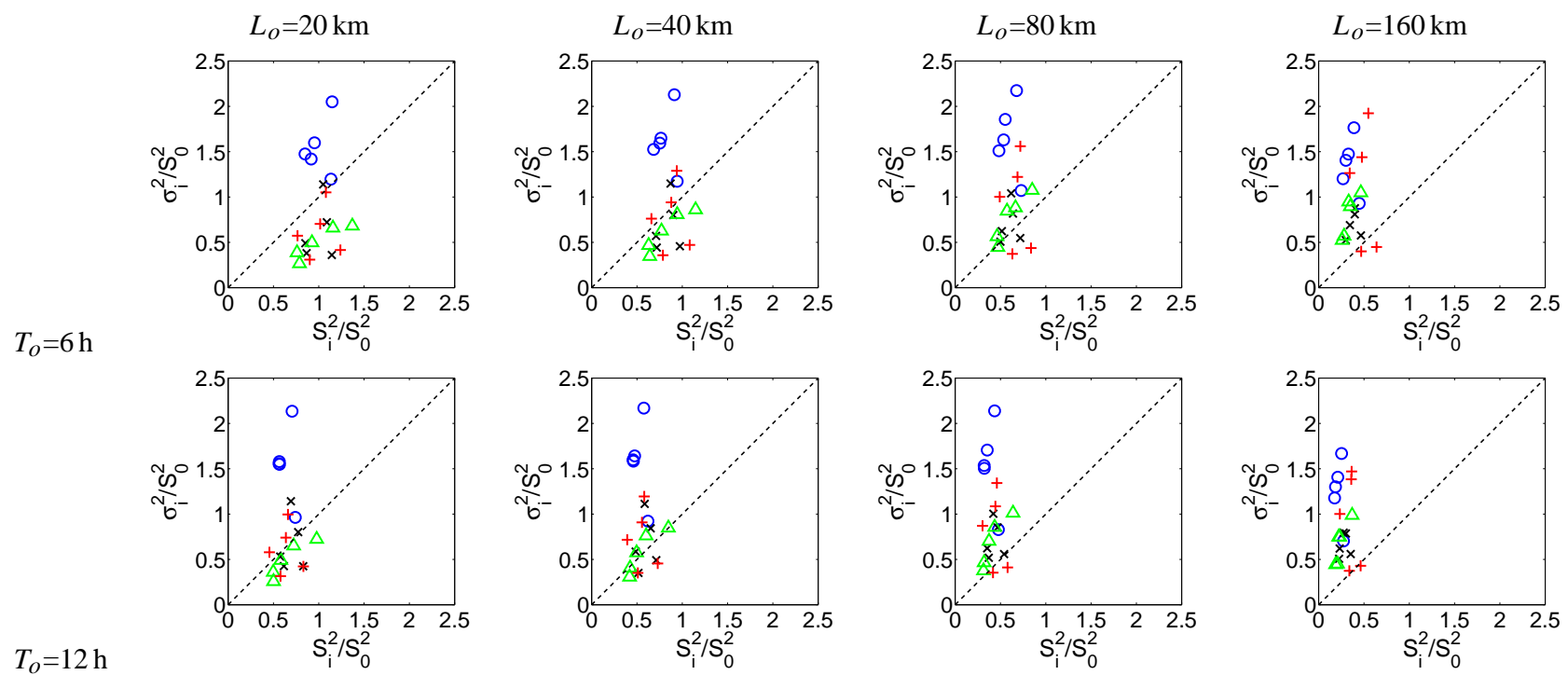

Fig. 3. Comparison of the width of each stochastic ensemble, $\sigma_{i}^{2}$, with its average distance from the other stochastic ensembles, $S_{i}^{2}$, for various choices of the reliability scales $L_{o}$ and $T_{o}$. Both measures are normalized by $\bar{S}_{o}^{2}$, the average spread of the dynamic ensemble at $L_{o}=20 \mathrm{~km}, T_{o}=6 \mathrm{~h}$. Each event is characterized by a different value of $\bar{S}_{o}^{2}$. Different symbols indicate the four events (case studies) considered (circles: 13 November 2002; x-crosses: 23 November 2002; triangles: 26 December 2002; plus signs: 20 January 2003). The dotted line indicates the 45 degree line for reference.
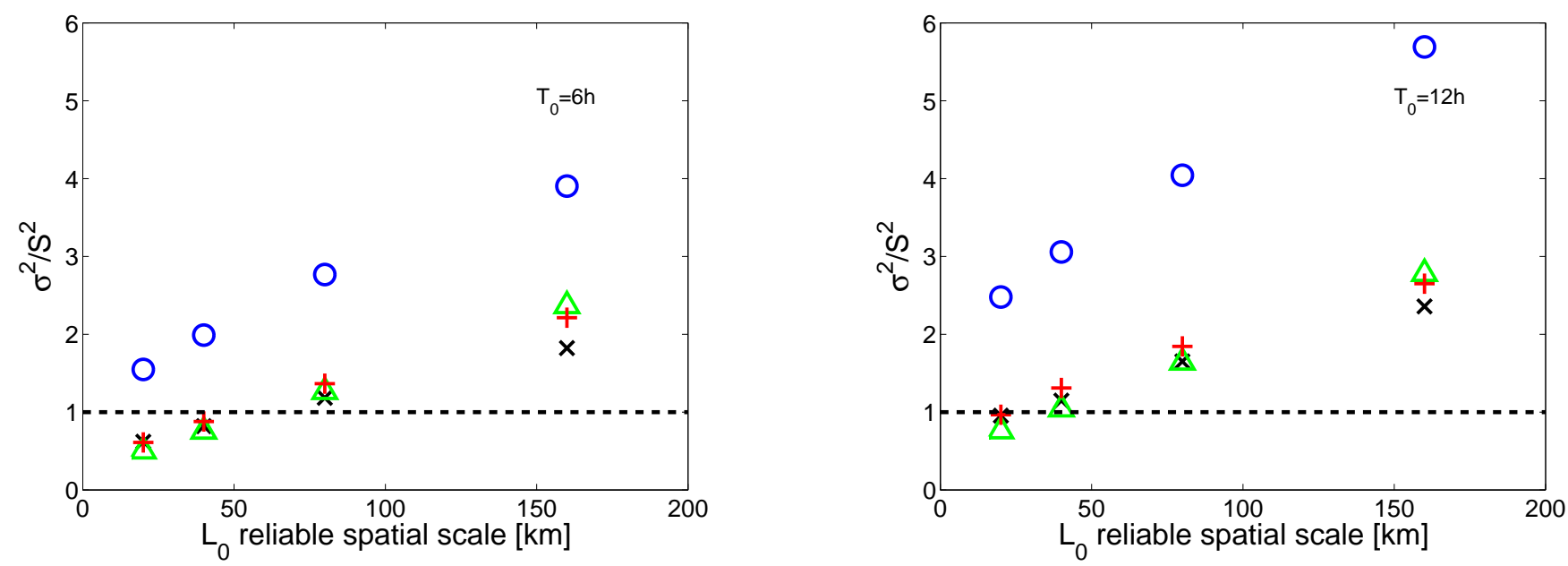

Fig. 4. Ratio between the average spread of stochastic ensembles, $\bar{\sigma}^{2}$, and the average spread of the dynamical LEPS ensemble, $\bar{S}^{2}$, as a function of the reliability scales $L_{o}$ and $T_{o}$. The symbols refer to the same events as described in Fig. 3.

are important for representing the total uncertainity. In one of the examples considered, the variance of the individual meteorological fields is so high that it leads to a spread of the stochastic ensemble which is definitely larger than the spread of the dynamical ensemble for all choices of the reliability scales. This suggests that there may not exist just one single choice of the reliability scales for all cases and that the correct choice could be conditioned on the properties of synoptic circulations. For an operational system, however, this would add an extra dimension of complexity.
The results reported above can help determining the components of the prediction chain where an improvement in uncertainty estimates would be most effective. Given that a dynamical forecasting system with a very large number of ensemble members with very high spatial and temporal resolution is currently unfeasible, the finite computational resources impose a trade-off between increasing small-scale resolution and enlarging the number of members in the dynamical ensemble. 
Numerical meteorological models and assimilation networks will presumably continue to improve in the future, possibly leading to smaller values of the reliability scales. In this case, the spread of the individual stochastic ensembles could become significantly smaller than the average distance between the different members of the dynamical ensemble. In such a scenario, ensemble stochastic downscaling and uncertainty below the resolved scales become less important; further effort should be put into the task of increasing the number of dynamical ensemble members and uncertainty estimates above the reliability scales. On the opposite, if a more detailed comparison between data and numerical predictions indicates that the reliability scales are significantly larger than the $40 / 60 \mathrm{~km}$ assumed here, most of the variability in the forecast would be associated with the stochastic downscaling procedure and with small-scale processes. In this case, increasing the number of dynamical ensemble members would be superfluous, and the effort should be put into achieving smaller, numerically reliable spatial and temporal scales.

The conclusions reported in this work rely on the assumption that the spread of the LEPS ensemble and the spread of the stochastic ensembles are correctly representing the uncertainity of the forecast respectively above and below the reliability scales. Indeed, COSMO-LEPS has been verified to provide satisfactory probabilistic forecasts of precipitation, particularly for intense events (Marsigli et al., 2005), and it can be considered appropriate for representing uncertainity above the reliability scales. Further, the RainFARM downscaling procedure has been shown to correctly reproduce the statistics of observed precipitation at small scales (Rebora et al., 2006b), and the spread of the stochastic ensembles can be considered to correctly represent uncertainity below the reliability scales.

Of course, stochastic downscaling does not capture the true physics of small-scale precipitation dynamics, and it simply propagates the information contained in large-scale forecasts. More insight could be gained from the analysis of a truly dynamical downscaling, i.e., by reaching smallscale resolution not by stochastic methods but by an ensemble of nested high-resolution numerical simulations. At the moment, however, this strategy seems difficult to follow in operational frameworks.

Acknowledgements. This work was supported by the project "Proscenio", funded by the Italian national Civil Protection Department. COSMO-LEPS is an operational system managed by ARPA-SIM, the Regional Hydro-Meteorological Service of Emilia-Romagna in Italy, which we thank for providing the data used in this work. The comments of the Editor and of two anonymous reviewers helped to improve the presentation of the results.

Edited by: O. Talagrand

Reviewed by: two referees

\section{References}

Adlerman, E. J. and Droegemeier, K. K.: The sensitivity of numerically simulated cyclic mesocyclogenesis to variations in model physical and computational parameters, Mon. Wea. Rev., 130, 2671-2691, 2002.

Bell, T. L.: A space-time stochastic model of rainfall for satellite remote sensing studies, J. Geophys. Res., 92, 9631-9643, 1987.

Buizza, R. and Palmer, T. N.: Impact of ensemble size on ensemble prediction, Mon. Wea. Rev., 126, 2503-2518, 1998.

Du, J., Mullen, S. L., and Sanders, F.: Short-range ensemble forecasting of quantitative precipitation, Mon. Wea. Rev., 125, 2427 2459, 1997.

Epstein, E. N.: Stochastic Dynamic Prediction, Tellus, 21, 739-759, 1969.

Ferraris, L., Rudari, R., and Siccardi, F.: The uncertainty in the prediction of flash floods in the northern Mediterranean environment, J. Hydrometeorol., 3, 714-727, 2002.

Ferraris, L., Gabellani, S., Rebora, N., and Provenzale, A.: A comparison of stochastic models for spatial rainfall downscaling, Water Resour. Res., 39, 1368, doi:10.1029/2003WR002504, 2003.

Frogner, I. L. and Iversen, T.: High-resolution limited-area ensemble predictions based on low-resolution targeted singular vectors, Quart. J. Roy. Meteorol. Soc., 128, 1321-1341, 2002.

Hamill, T. M. and Colucci, S. J.: Verification of Eta-RSM shortrange ensemble forecasts, Mon. Wea. Rev., 125, 1312-1327, 1997.

Hamill, T. M. and Colucci, S. J.: Evaluation of Eta-RSM ensemble probabilistic precipitation forecasts, Mon. Wea. Rev., 126, 711724, 1998.

Leith, C. E.: Theoretical skill of Monte Carlo forecasts, Mon. Wea. Rev., 102, 409-418, 1974.

Lovejoy, S. and Mandelbrot, B. B.: Fractal properties of rain, and a fractal model, Tellus, 37 A, 209-232, 1985.

Marsigli, C., Montani, A., Nerozzi, F., Paccagnella, T., Tibaldi, S., Molteni, F., and Buizza, R.: A strategy for high-resolution ensemble prediction. II: Limited-area experiments in four Alpine flood events, Quart. J. Roy. Meteorol. Soc., 127, 2095-2115, 2001.

Marsigli, C., Montani, A., Nerozzi, F., and Paccagnella, T.: Probabilistic high-resolution forecast of heavy precipitation over Central Europe, Nat. Hazards Earth Syst. Sci., 4, 315-322, 2004, http://www.nat-hazards-earth-syst-sci.net/4/315/2004/.

Marsigli, C., Boccanera, F., Montani, A., and Paccagnella, T.: The COSMO-LEPS mesoscale ensemble system: validation of the methodology and verification, Nonlin. Processes Geophys., 12, 527-536, 2005, http://www.nonlin-processes-geophys.net/12/527/2005/.

Menabde, M., Seed, A., Harris, D., and Austin, G.: Self-similar random fields and rainfall simulations, J. Geophys. Res., 102D, 13 509-13 515, 1997.

Molteni, F., Buizza, R., Palmer, T. N., and Petroliagis, T.: The ECMWF ensemble prediction system: Methodology and validation, Quart. J. Roy. Meteorol. Soc., 122, 73-119, 1996.

Molteni, F., Buizza, R., Marsigli, C., Montani, A., Nerozzi, F., and Paccagnella, T.: A strategy for high-resolution ensemble prediction. I: Definition of representative members and global-model experiments, Quart. J. Roy. Meteorol. Soc., 127, 2069-2094, 2001.

Montani, A., Marsigli, C., Nerozzi, F., Paccagnella, T., and Buizza, 
R.: Performance of the ARPA-SMR limited-area ensemble prediction system: two flood cases, Nonlin. Processes Geophys., 8, 387-399, 2001, http://www.nonlin-processes-geophys.net/8/387/2001/.

Montani, A., Marsigli, C., Nerozzi, F., Paccagnella, T., Tibaldi, S., and Buizza, R.: The Soverato flood in Southern Italy: performance of global and limited-area ensemble forecasts, Nonlin. Processes Geophys., 10, 261-274, 2003, http://www.nonlin-processes-geophys.net/10/261/2003/.

Palmer, T.: Predicting uncertainty in forecasted weather and climate, Rep. Prog. Physics, 125, 71-116, 2000.

Palmer, T. N.: Extended-range atmospheric prediction and the Lorenz model, Bull. Am. Meteorol. Soc., 74, 49-65, 1993.

Palmer, T. N.: A nonlinear dynamical perspective on model error: A proposal for non-local stochastic-dynamic parametrization in weather and climate prediction models, Quart. J. Roy. Meteorol. Soc., 127, 279-304, 2001.

Patterson, G. and Orszag, S.: Spectral calculations of isotropic turbulence: efficient removal of aliasing interaction, Phys. Fluids, 14, 2538-2541, 1971.

Perica, S. and Foufoula-Georgiu, E.: Model for multiscale disaggregation of spatial rainfall based on coupling meteorological and scaling descriptions, J. Geophys. Res., 101D, 26347-26 361, 1996.
Rebora, N., Ferraris, L., von Hardenberg, J., and Provenzale, A.: Rainfall downscaling and flood forecasting: a case study in the Mediterranean area, Nat. Hazards Earth Syst. Sci., 6, 611-619, 2006a.

Rebora, N., Ferraris, L., von Hardenberg, J., and Provenzale, A.: RainFARM: Rainfall downscaling by a filtered autoregressive model, J. Hydrometeorol., 7, 724-738, 2006b.

Roulston, M. S. and Smith, L. A.: Combining dynamical and statistical ensembles, Tellus Series A-Dynamic Meteorology And Oceanography, 55, 16-30, 2003.

Sattler, K. and Feddersen, H.: Limited-area short-range ensemble predictions targeted for heavy rain in Europe, Hydrol. Earth Syst. Sci., 9, 300-312, 2005, http://www.hydrol-earth-syst-sci.net/9/300/2005/.

Siccardi, F., Boni, G., Ferraris, L., and Rudari, R.: A hydrometeorological approach for probabilistic flood forecast, J. Geophys. Res.-Atmos., 110, D05101, doi:10.1029/2004JD005314, 2005.

Toth, Z. and Kalnay, E.: Ensemble forecasting at NMC - the generation of perturbations, Bull. Am. Meteorol. Soc., 74, 2317-2330, 1993.

Toth, Z. and Kalnay, E.: Ensemble forecasting at NCEP and the breeding method, Mon. Wea. Rev., 125, 3297-3319, 1997.

Waymire, E., Gupta, V., and Rodriguez-Iturbe, I.: A spectral theory of rainfall intensity at the meso-beta scale, Water Resour. Res. 20, 1453-1465, 1984. 